\title{
3D scattering by multiple cylindrical cavities buried in an elastic formation
}

\author{
Julieta Antonio, Antonio Tadeu* \\ University of Coimbra, Department of Civil Engineering, 3000-000 Coimbra, Portugal
}

(Received 9 March 2000; revised and accepted 21 September 2000)

\begin{abstract}
This paper presents the three-dimensional scattering field obtained when multiple cylindrical circular cavities of infinite length buried in a homogeneous elastic medium, are subjected to dilatational point loads placed at some point in the medium. The solution is formulated using boundary elements for a wide range of frequencies and spatially harmonic line loads, which are then used to obtain time series by means of (fast) inverse Fourier transforms into space-time. The method and the expressions presented are implemented and validated by applying them to a cylindrical circular cavity buried in an infinite homogeneous elastic medium subjected to a dilatational point load, for which the solution is calculated in closed form. The boundary elements method is then used to evaluate the wave-field elicited by a dilatational point load source in the presence of a different number of cylindrical oval cavities. Simulation analyses using this idealized model are then applied to the study of wave propagation patterns in the vicinity of these inclusions. The amplitude of the wavefield in the frequency vs axial-wavenumber domain is presented, allowing the recognition, identification, and physical interpretation of the variation of the wavefield. (C) 2001 Éditions scientifiques et médicales Elsevier SAS
\end{abstract}

3D wave scattering / multiple cavities / elastic medium

\section{Introduction}

The propagation of waves between a source and a receiver placed at depth below the ground surface, in the vicinity of elastic inclusions and topographical deformations, has occupied many researchers for years. Some of the first analytical studies on wave diffraction and scattering looked at wave motion and reverberations in alluvial basins of regular shape (Trifunac, 1971; Wong and Trifunac, 1974), and the wave scattering induced by cavities (Lee, 1977, 1988; Datta and Shah, 1982; Lee and Karl, 1992). More recently, semi-analytical methods have been used to study wave diffraction caused by geological irregularities of arbitrary shape within globally homogeneous media (Sanchez-Sesma, 1983; Moeen-Vaziri and Trifunac, 1988; Lee and Wu, 1994). By contrast, the application of purely numerical methods (i.e. finite elements or differences combined with boundaries) have mostly been employed in situations where the response is required only within localised irregular domains, such as soil-structure interaction problems (Waas, 1972; Lysmer et al., 1974; Kausel, 1974). Discrete methods have also occasionally been used to model large alluvial basins, but only in planestrain (Ohtsuki and Harumi, 1983). Finally, hybrid methods involving a combination of finite elements to model the interior domain containing the inhomogeneities and semi-analytical representations for the exterior domain have been used (Shah et al., 1982). These methods have mostly been employed in cases where a twodimensional (2D) solution is wanted. If the full scattering wave field generated by sources placed in the presence of three-dimensional (3D) propagation media is evaluated, then the numerical calculations are very costly in terms of computer effort.

If the medium is $2 \mathrm{D}$ and the dynamic source 3D (such as a point load), often termed a two-and-a-halfdimensional problem (2-1/2-D), the solution becomes much simpler. Solutions for such problems involve a two

* Correspondence and reprints: tadeu@dec.uc.pt 
spatial Fourier transform in the direction in which the geometry does not vary. A sequence of 2D problems with different spatial wavenumbers, $k_{z}$, must first be solved, and then the inverse Fourier transform is used to synthesize the 3D field.

The 2-1/2-D wave equation for inclusions such as the circular cylinder can be separated, and so their solution can be known in closed form. The solution is harder to determine for inclusions with irregular cross-sections. In these cases, the Boundary Element Method (BEM) may be the best tool, especially if the solution has to satisfy the far field conditions required if the inclusions are buried in an unbounded or half-space elastic medium. Stamos and Beskos (1996) used the BEM to describe the 3D dynamic response of long lined tunnels with a uniform cross-section, buried in a half-space, to plane harmonic waves propagated in an arbitrary direction, treating it as a two-dimensional problem. Pedersen et al. (1994) studied the three-dimensional seismic response of two-dimensional topographies to plane waves by means of the indirect boundary element method (IBEM), making use of the Green's functions for a harmonic point force moving along the axis of the topography in a full space.

In this work, the BEM is used to evaluate the wave-field elicited by a dilatational point load (blast load) in the presence of irregular cylindrical inclusions buried in an unbounded elastic medium. The solution at each frequency is given in terms of waves with varying wavenumber, $k_{z}$ (where $z$ is the direction in which the geometry does not vary). This is then Fourier transformed into the spatial domain. To find the wavenumber transform in discrete form, it is assumed that an infinite number of virtual point sources are equally spaced along the $z$ axis, far enough apart to prevent spatial contamination (Bouchon and Aki, 1977). The analyses utilize complex frequencies, moving down the frequency axis, in the complex plane. Any singularities on or close to the axis are thus eliminated and the neighboring fictitious sources can only exert a very slight influence (Phinney, 1965).

The BEM method is first formulated and validated by applying it to a cylindrical circular cavity buried in an infinite homogenous elastic medium subjected to a dilatational point load for which the solution is calculated in closed form. This model is further used for simulation analyses to investigate wave propagation in the vicinity of such inclusions, in the presence of nearby, varying-sized, cylindrical oval cavities. Results are obtained in the frequency and time domains, in particular for different apparent wave velocities along the $z$ axis, to quantitatively study the 3D effects of the scattering. Many simulations were performed to study how different parameters, such as the size of the inclusions, affect the three-dimensional scattering wave patterns.

\section{Problem formulation}

An infinitely long cylindrical irregular cavity is buried within a spatially uniform elastic medium (figure 1) and subjected to a harmonic dilatational point source at position $\left(x_{0}, 0,0\right)$, oscillating with a frequency $\omega$. The incident field can be expressed by means of the now classical dilatational potential $\phi$

$$
\phi_{\text {inc }}=\frac{A \mathrm{e}^{i \frac{\omega}{\alpha}\left(\alpha t-\sqrt{\left(x-x_{0}\right)^{2}+y^{2}+z^{2}}\right)}}{\sqrt{\left(x-x_{0}\right)^{2}+y^{2}+z^{2}}},
$$

where the subscript inc denotes the incident field, $A$ is the wave amplitude, $\alpha$ is the compressional wave velocity of the medium, and $i=\sqrt{-1}$.

Defining the effective wavenumbers

$$
k_{\alpha}=\sqrt{\frac{\omega^{2}}{\alpha^{2}}-k_{z}^{2}}, \quad \operatorname{Im} k_{\alpha}<0
$$




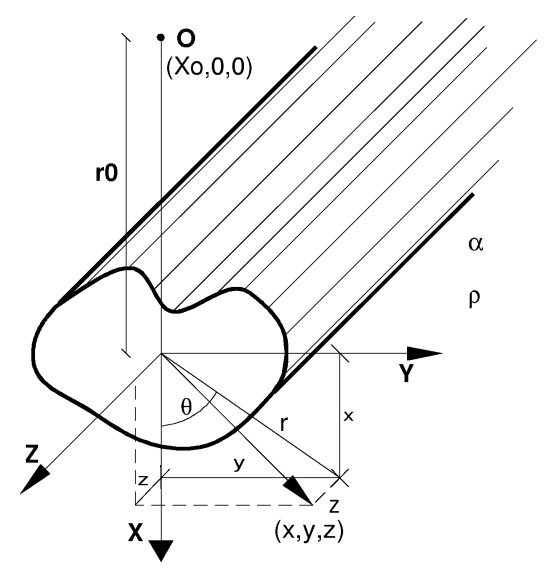

Figure 1. Geometry of the problem.

by means of the axial wavenumber $k_{z}$, and Fourier-transforming equation (1) in the $z$ direction, we get

$$
\hat{\phi}_{\mathrm{inc}}\left(\omega, x, y, k_{z}\right)=\frac{-i A}{2} H_{0}^{(2)}\left(k_{\alpha} \sqrt{\left(x-x_{0}\right)^{2}+y^{2}}\right)
$$

in which the $H_{n}^{(2)}(\cdots)$ are second Hankel functions of order $n$.

When we take an infinite number of virtual point sources, spaced at equal intervals along the $z$ direction at a sufficient distance, $L$, from each other to avoid spatial contamination (Bouchon and Aki, 1977), the incident field may be written as

$$
\phi_{\mathrm{inc}}(\omega, x, y, z)=\frac{2 \pi}{L} \sum_{m=-\infty}^{\infty} \hat{\phi}_{\mathrm{inc}}\left(\omega, x, y, k_{z}\right) \mathrm{e}^{-i k_{z m} z}
$$

with $k_{z m}=\frac{2 \pi}{L} m$. This equation converges and can be approximated by a finite sum of terms.

\section{Boundary element formulation}

The 3D field generated by a cylindrical cavity subjected to spatially sinusoidal harmonic line loads (defined by equation (3)) is found by means of the Boundary Element Method (BEM).

Since the basic equations used are known (Manolis and Beskos, 1988 and Banerjee, 1994), a detailed explanation here is unnecessary. The boundary integral equations, in the absence of distributed loads and in the presence of virtual point loads, $\delta\left(x-x_{0}\right)$, leads to the following equation,

$$
c_{i j} u_{j}\left(x_{0}, \omega\right)=\int_{C} t_{i}(x, v, \omega) G_{i j}\left(x, x_{0}, \omega\right) \mathrm{ds}-\int_{C} H_{i j}\left(x, v, x_{0}, \omega\right) u_{j}(x, \omega) \mathrm{ds},
$$

where $i, j=1,2$ represents the normal and tangential directions in relation to the cavity surface; $i, j=3$ indicates the $z$ direction, $G_{i j}\left(x, x_{0}, \omega\right), H_{i j}\left(x, v, x_{0}, \omega\right)$ are the displacements and tractions in the direction $j$ at $x$, on boundary $C$, caused by a unit point force applied at the source, $x_{0}$, in direction $i$; vector $v$ is the unit outward normal at the boundary, and $c_{i j}$ is a constant that equals $\delta_{i j} / 2$ for a smooth boundary, where Kronecker's delta is represented by $\delta_{i j}$. 
Two-and-a-half Green's functions are used to determine the expressions for the tensions. The strains are calculated from partial derivatives of the fundamental two-and-a-half dimension solution, and Hooke's law is subsequently applied to find the stresses. Appendix A gives details of the displacement and stress functions applicable to the present problem, in the form of Cartesian co-ordinates. Equilibrium relations are used to transform the stress fields into the normal, tangential and local $(z)$ co-ordinate systems at each element. Since the boundary conditions for a cavity impose null tractions, a simplified form of equation (5) is obtained:

$$
c_{i j} u_{j}\left(x_{0}, \omega\right)=-\int_{C} H_{i j}\left(x, v, x_{0}, \omega\right) u_{j}(x, \omega) \mathrm{ds}
$$

The boundary and boundary values must be discretized before this integral can be evaluated for an arbitrary cross-section. Therefore, in a situation where $N$ boundary elements are used and nodal displacements within each element are taken to be constant and to have the same value at the respective nodal point, equation (6) becomes,

$$
c_{i j} u_{j}^{k}=-\sum_{n=1}^{N} H_{i j}^{k n} u_{j}^{n} .
$$

In equation (7), the element number at the point where the virtual load is applied is represented by $k$, the boundary values in element $n$ are represented by $u_{j}^{n}$, and $H_{i j}^{k n}$ signifies the element integrals

$$
H_{i j}^{k n}=\int_{C_{n}} H_{i j}\left(x_{n}, v_{n}, x_{k}, \omega\right) \mathrm{ds},
$$

where $v_{n}$ is the unit outward normal for $n$th boundary segment $C_{n}$.

If each node on the boundary is subjected to a virtual load we obtain a system of linear equations that relates nodal forces and nodal displacements. These equations can be solved for the nodal displacements.

When an incident wave strikes the cavity, equation (6) is changed as follows,

$$
c_{i j} u_{j}\left(x_{0}, \omega\right)=-\int_{C} H_{i j}\left(x, v, x_{0}, \omega\right) u_{j}(x, \omega) \mathrm{ds}+u_{j}^{\mathrm{inc}}\left(x_{0}, \omega\right)
$$

To determine the incident field in this equation, we take partial derivatives of equation (3) and calculate the displacements along $x, y$ and $z$ and then apply equilibrium relations, which gives the normal and tangential displacements along the boundary.

If the displacements and tractions are allowed to vary linearly within the boundary elements, the required integrals of equation (8) change to

$$
H_{i j}^{k n}=\int_{C_{n}} \phi H_{i j}\left(x_{n}, v_{n}, x_{k}, \omega\right) \mathrm{ds},
$$

where $\phi$ represents the linear interpolation functions.

The displacement and stress variations within a boundary element are defined in terms of the nodal values. The traction discontinuity at the corner between two boundary elements is handled by means of discontinuous boundary elements. Thus, the nodes that would meet at the corner are moved inside (Brebbia et al., 1984).

After mathematical manipulation of the integral equations, combined and subjected to the continuity conditions at the interface between the two media, and discretized appropriately, a system of equations is 
obtained that can be solved for the nodal displacements. The required integrations in equation (10) are performed using Gaussian quadrature when the element to be integrated is not the loaded element. For the loaded element, the existing singular integrands are carried out in closed form (Tadeu et al., 1999-a, 1999-b).

\section{Displacements in space-time}

A numerical fast Fourier transform in $k_{z}$ gives the displacements in the spatial-temporal domain, taking a source whose temporal variation is given by a Ricker wavelet, as defined below. The Ricker wavelet has the advantage of decaying rapidly, in both time and frequency, which both reduces computational effort, and allows the computed time series and synthetic waveforms to be interpreted more easily.

The Ricker wavelet function is given by

$$
u(\tau)=A\left(1-2 \tau^{2}\right) \mathrm{e}^{-\tau^{2}},
$$

where $A$ is the amplitude, $T=\left(t-t_{s}\right) / t_{o}$ and $t$ denotes time; $t_{s}$ is the time when the maximum occurs, while $\pi t_{o}$ is the characteristic (dominant) period of the wavelet. Its Fourier transform is

$$
U(\omega)=A\left[2 \sqrt{\pi} t_{o} \mathrm{e}^{-i \omega t_{s}}\right] \Omega^{2} \mathrm{e}^{-\Omega^{2}}
$$

in which $\Omega=\omega t_{o} / 2$.

The Fourier transformations are achieved by discrete summations over wavenumbers and frequencies, which is mathematically the same as adding periodic sources at spatial intervals $L=2 \pi / \Delta k_{z}$ (in the $z$-axis), and temporal intervals $T=2 \pi / \Delta \omega$, with $\Delta k_{z}$, and $\Delta \omega$ being the wavenumber and frequency steps, respectively (Bouchon and Aki, 1977). The spatial separation $L$ must be large enough so that contamination of the response by the periodic sources does not occur. Thus, the contribution to the response by the fictitious sources must

occur at times later than $T$. It is also very useful for this if the frequency axis is shifted slightly downward, by considering complex frequencies with a small imaginary part of the form $\omega_{c}=\omega-i \eta$ (with $\eta=0.7 \Delta \omega$ ). The periodic sources are thus practically eliminated. In the time domain, this shift is later taken into account by applying an exponential window $\mathrm{e}^{\eta t}$ to the response (Kausel and Roesset, 1992).

\section{Validation of the BEM algorithm}

The BEM algorithm was implemented and validated by applying it to a cylindrical circular cavity, placed in a homogeneous elastic medium, subjected to a dilatational point load applied at point $\mathrm{O}$, as in figure 2, for which the solution is known in closed form and described in Eringen (1961) and Pao and Mow (1973).

The response is calculated over a fine vertical grid plane, placed perpendicular to the axis. Figure 3 gives the horizontal scattered displacement field computed when a harmonic pressure load of $2450 \mathrm{~Hz}$ is excited. This figure gives both the response obtained with the closed form solution (figure 3a), and the difference in the response obtained using the BEM, the error, when the inclusion is modelled with 17 and 52 boundary elements, figures $3 b$ and $3 c$, respectively.

In the present example, the ratio between the wavelength of the shear waves to length of boundary elements varies between 3 (17 boundary elements) and 9.0 (52 boundary elements). The BEM accuracy improves as shorter boundary elements are used to model the response, as anticipated. 


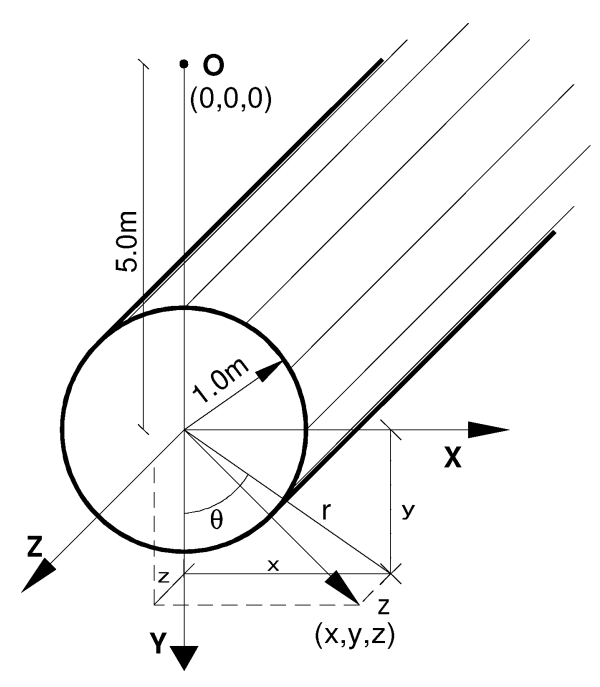

$$
\begin{gathered}
\alpha=4208 \mathrm{~m} / \mathrm{s} \\
\beta=2656 \mathrm{~m} / \mathrm{s} \\
\rho=2140 \mathrm{Kg} / \mathrm{m}^{3}
\end{gathered}
$$

Figure 2. Circular cylindrical inclusion in an unbounded elastic medium.

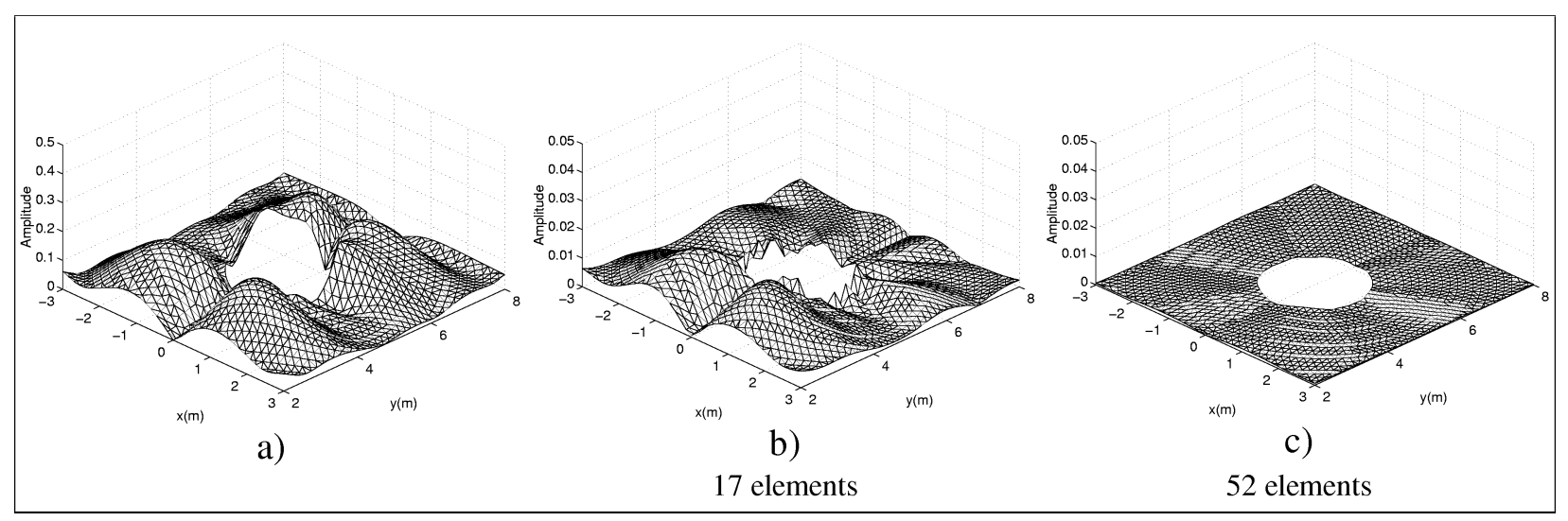

Figure 3. Horizontal scattered displacement field for cylindrical circular inclusion in unbounded medium: (a) Analytical solution; (b) BEM error $(\lambda / L)=3 ;($ c) BEM error $(\lambda / L)=9$.

\section{Numerical applications}

Simulation analyses were performed when two cylindrical, oval buried cavities, placed inside an elastic unbounded medium are both illuminated by an incident blast load defined by the dilatational $\phi$ expressed as in equation (1). Four differently-sized inclusions are considered, defined by their perimeter $(2 \pi R \mathrm{~m})$. In this equation $R$ is a parameter that takes the values of $R=0.0 \mathrm{~m}, 2.5 \mathrm{~m}, 5.0 \mathrm{~m}$ and $10.0 \mathrm{~m}$. One of the cavities is kept constant $(R=10.0 \mathrm{~m})$ while the other, placed in its vicinity, changes in size. Figure 4 displays the geometry of the cross-section of the inclusions considered here. At time $t=0$, a point source at a point $\mathrm{O}$ creates a spherical dilatational pulse propagating away from $\mathrm{O}$. The field generated is computed at 41 receivers, spaced at equal intervals $(2.0 \mathrm{~m})$ along the $x$ direction.

The dilatational wave velocity $(\alpha=4208 \mathrm{~m} / \mathrm{s})$, the shear wave velocity $(\beta=2656 \mathrm{~m} / \mathrm{s})$ and density $\left(\rho=2140 \mathrm{Kg} / \mathrm{m}^{3}\right)$ of the host elastic medium are kept constant in all the analyses. The computations are performed in the frequency range $(15,960 \mathrm{~Hz})$, with a frequency increment of $15.0 \mathrm{~Hz}$, which determines the 
total time duration ( $T=66.6 \mathrm{~ms}$ ) for the analysis in the time domain. The spatial distance between virtual point sources has been set to $561.0 \mathrm{~m}=2 \alpha T$. The source time dependence is a Ricker wavelet with a characteristic frequency of $320 \mathrm{~Hz}$.

The oval cavities are modelled with boundary elements, the number of which changes with the frequency of excitation of the harmonic load. A ratio between the wavelength of the incident waves to length of the boundary elements is kept at a minimum of 8.0. In any case the minimum number of the boundary elements used to model each cavity is less than 30 .

Figure 5 displays the three dimensional vertical $(y)$ time displacements (0-30 ms) computed for all receivers when only the first cavity is present $(R=10.0 \mathrm{~m})$. In addition, the amplitude in the frequency vs. axialwavenumber domain of these responses are presented for the two receivers placed closest to, and farthest from the cavity, respectively. Each plot is normalized with respect to its own maximum. The time records exhibit non-dispersive body waves and guided waves. The first set of pulses recorded at the receivers corresponds to the incident field, second arrivals are caused by the initial $\mathrm{P}$ waves reflected from the cavity, while third arrivals are $\mathrm{S}$ mode converted waves resulting from the $\mathrm{P}$ wave incidence on the cavity. These pulses are identified in this figure with the labels $\mathrm{I}, \mathrm{P}_{1}$ and $\mathrm{S}_{1}$, respectively.

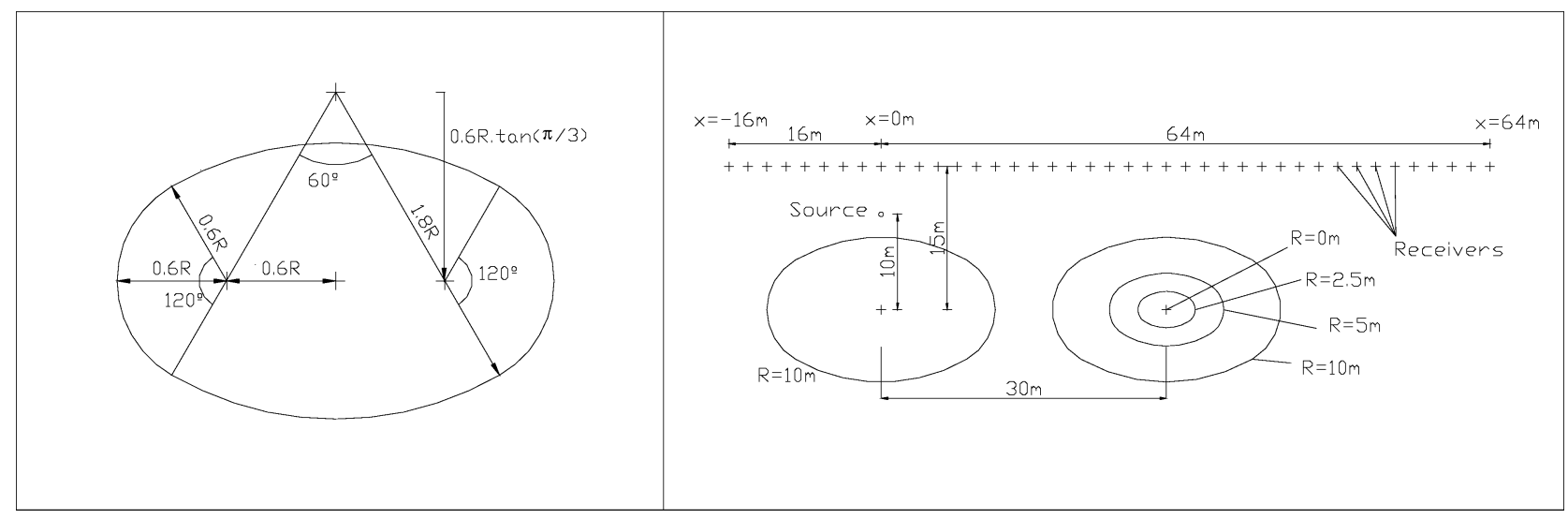

Figure 4. Geometries of the numerical application problems.

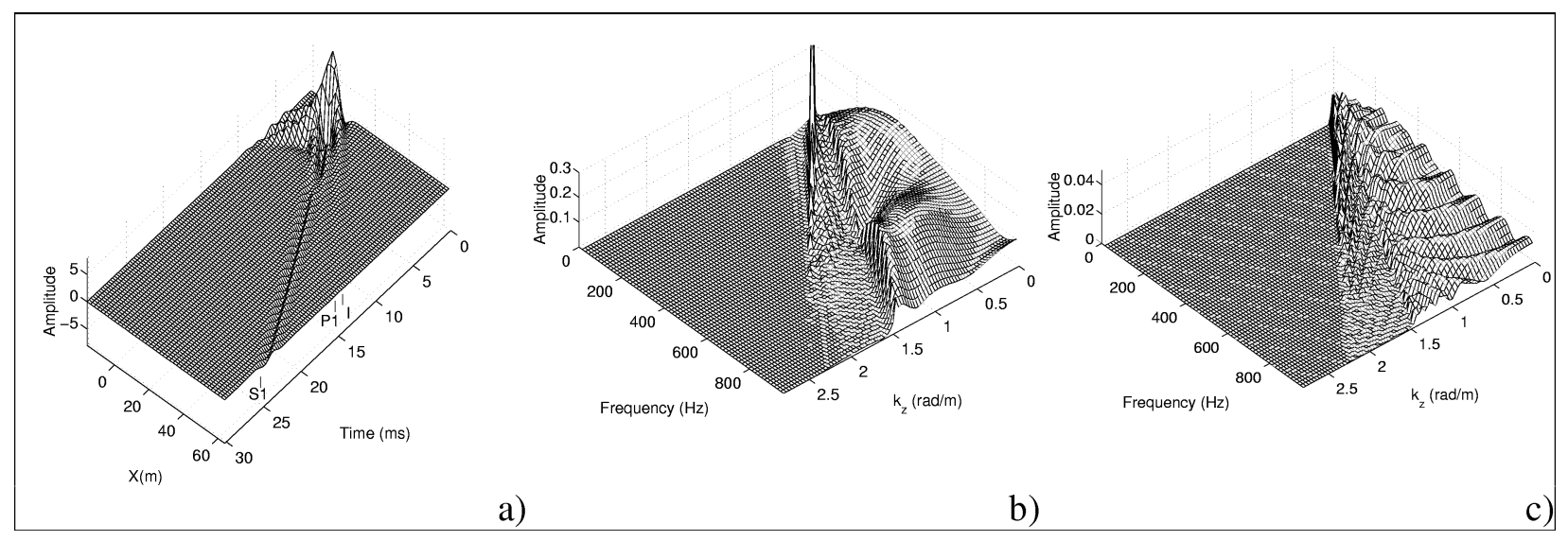

Figure 5. Three Dimensional vertical response when only one cavity is present $(R=10.0 \mathrm{~m})$ : (a) time response; (b) Fourier spectra response at receiver $\# 9$; (c) Fourier spectra response at receiver \#41. 
The guided waves propagating along the surface of the cavity decay very rapidly with the increasing distance to its surface. The Fourier spectra of the responses allow a visual separation of the different wave types. Waves travelling with different velocities $(c)$ appear in these plots at different positions, creating wrinkles accordingly to $\omega / c$. As an example, at $f=960.0 \mathrm{~Hz}$, the waves travelling with a $\mathrm{P}$ and $\mathrm{S}$ wave velocities, appears at $k_{z}=\omega / c=1.43 \mathrm{rad} / \mathrm{m}$ and $k_{z}=2.27 \mathrm{rad} / \mathrm{m}$, respectively. Analysis of these plots allow us to verify that the guided waves are important in the vicinity of the cavity and at lower frequencies.

The full three dimensional results, displayed in figure 5, required high computational effort given the large number of 2D solutions that needed to be integrated. Next, simulations are performed, following waves with different apparent wave velocities along the $z$ axis, to tackle this problem while seeking the main features of the 3D effects of the scattering. This apparent wave velocity $(c)$ results from waves arriving at the $z$ axis with a path inclination given by $\arccos \left(\frac{v}{c}\right)$, where $v$ is the true wave velocity (see figure 6 ). Thus, in the equations presented above, $k_{z}$ is taken to be $\omega / c$. In the examples selected, four apparent velocities $(c)$ are chosen, namely $c=\infty \mathrm{m} / \mathrm{s}, c=7000 \mathrm{~m} / \mathrm{s}, c=4208 \mathrm{~m} / \mathrm{s}$ and $c=2656 \mathrm{~m} / \mathrm{s} . c=\infty \mathrm{m} / \mathrm{s}$ corresponding to waves arriving at the receivers with a $90^{\circ}$ inclination in relation to the $z$ axis, which can be understood as a pure two-dimensional problem where the source is linear. As the path inclination ranges from $90^{\circ}$ to $0^{\circ}$, there is a lower bound value for $c$, which corresponds to the slowest wave velocities. As the frequency tends to the infinite the velocity of these waves approaches the Rayleigh wave velocity (Bamberger et al., 1991). Below this value, there are inhomogeneous waves which decay very fast with decreasing values of $c$.

Figure 7 again displays the vertical $(y)$, the $x$ and $z$ total displacement time responses recorded at the receivers when only one cavity is present $(R=10.0 \mathrm{~m})$, for different apparent wave velocities. Figure $7 a$ shows the response when $c=\infty \mathrm{m} / \mathrm{s}$. In this figure, the incident pulse, the $\mathrm{P}$ reflected pulse from the cavity, the $\mathrm{S}$ converted pulse are labelled $\mathrm{I}, \mathrm{P}_{1}$ and $\mathrm{S}_{1}$, respectively. The arrival times obtained for the different pulses are consistent with predictions by ray acoustics. A geometric acoustic ray analysis shows that these signals result from the propagation of a reflected and diffracted wavefront on the surface of the inclusion. A ray is associated with each wavefront, and thus there are two principal rays backscattered from such an inclusion. Figure 8 illustrates the propagation path of these waves between the source and the receiver.

As the apparent velocity decreases, both the arrival times of the different pulses and their amplitude decrease (see figures $7 a$ to $7 d$ ), indicating that the scattering energy is mainly concentrated in the vertical $z$ plane containing the dynamic source. A pulse in these plots, with a travel time $\tau$, corresponds to waves that travel from the source to the reflector and back to the receiver, along the same ray path inclination in relation to the $z$ axis. The travel distance $(L)$ in this domain is smaller because it corresponds to the projection of the initial vertical path $(d)$ to the inclined path, leading to a distance $L=d \sin \left[\arccos \left(\frac{v}{c}\right)\right]$. In this way, a fall in the apparent velocity causes a better separation of the P, S and guided waves (see figure $7 b$ ). Thus, when the apparent velocity equals the velocity of the $\mathrm{P}$ waves (see figure $7 c$ ), the waves travelling with this velocity arrive at the various receivers at $t=0.0$, and only the waves travelling with lower velocity, the $\mathrm{S}$ and guided

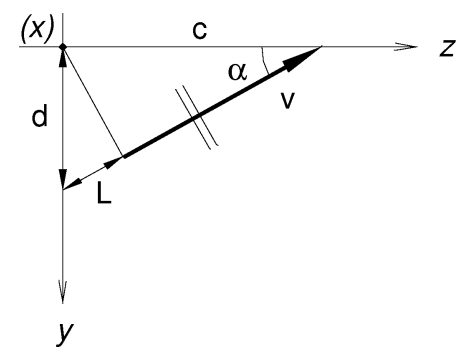

Figure 6. Apparent wave velocity. 


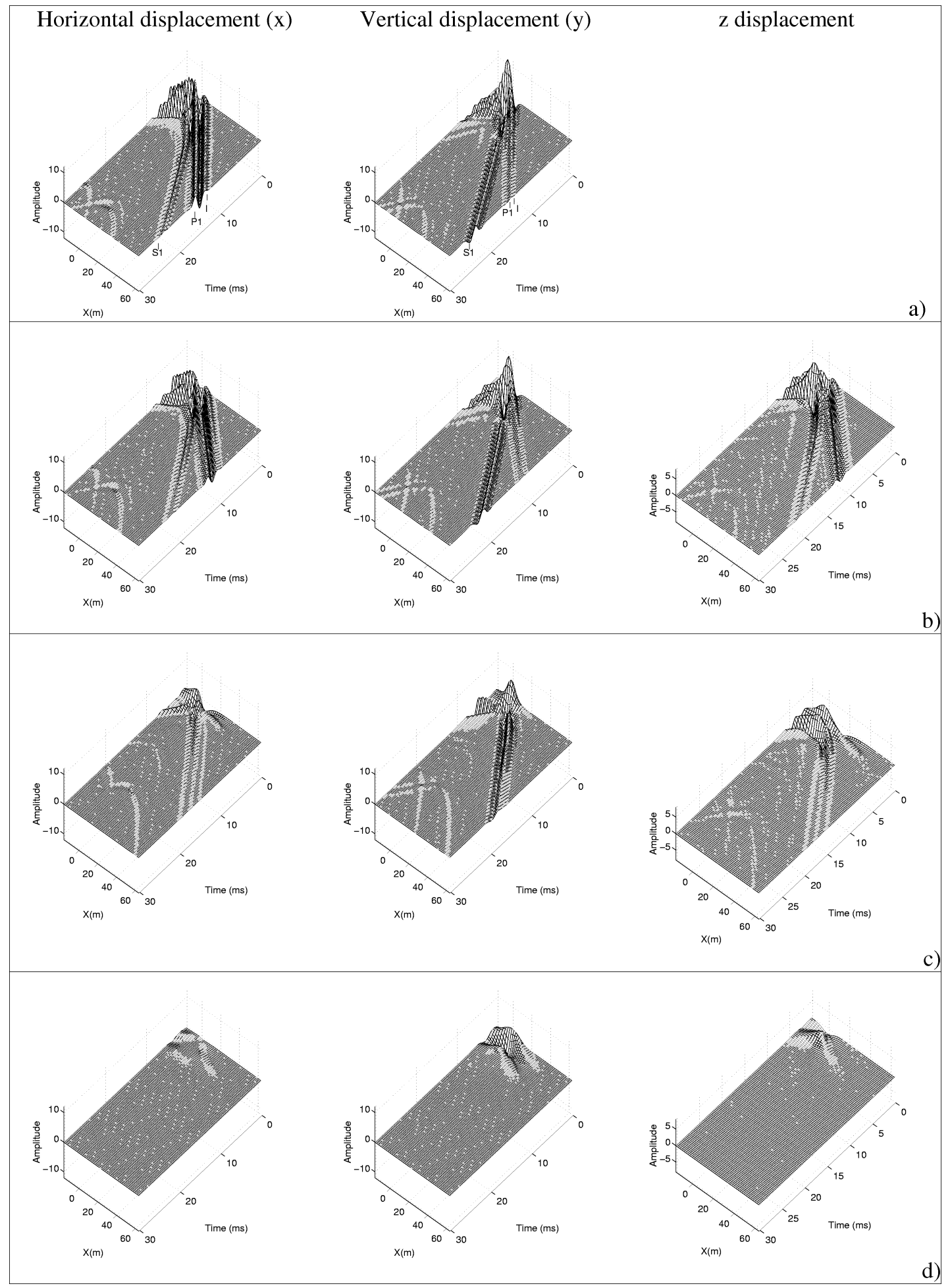

Figure 7. Synthetic seismograms when only one cavity is present $(R=10.0 \mathrm{~m}):$ (a) $c=\infty \mathrm{m} / \mathrm{s} ;$ (b) $c=7000 \mathrm{~m} / \mathrm{s} ;$ (c) $c=4208 \mathrm{~m} / \mathrm{s} ;$ (d) $c=2656 \mathrm{~m} / \mathrm{s}$. 


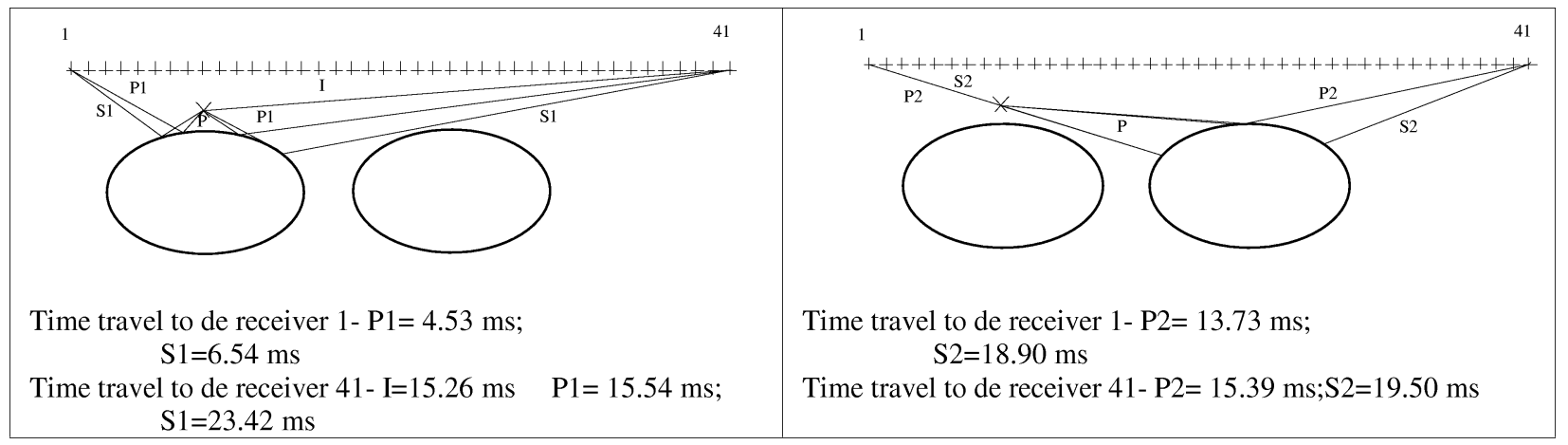

Figure 8. Propagation path of incident and reflected waves travelling between source $\mathrm{O}$ and the receivers 1 and 41 .

waves, survive in these time plots. Furthermore, when the apparent velocity is assumed to be $c=2656 \mathrm{~m} / \mathrm{s}$, only the guided waves survive. Our results confirm that the guided waves are only important in the vicinity of the inclusion (see figure 7d). As expected, the vertical displacement of the guided waves is larger than its $x$ displacement.

Figure 9 depicts the total vertical displacements when a second cavity, assuming three different sizes ( $R=10.0 \mathrm{~m}, 5.0 \mathrm{~m}$ and $2.5 \mathrm{~m})$, is added. In these plots, $c$ changes from $\infty$ to $2656 \mathrm{~m} / \mathrm{s}$. When $c=\infty \mathrm{m} / \mathrm{s}$, the first wave arrivals observed in figure $9 a$ correspond to the incident waves followed by wave trains that are directly reflected by each of the cavities. These are succeeded by pulses of progressively lower amplitude, which are the result of reverberations between the cavities. The time-of-flight of each of these pulses corresponds to the travel path of a pulse from the source to the inclusion and back to the receivers. Hence, the ray theory can be used to ascertain the accuracy of the numerical prediction, at least as far as arrival times of pulses are concerned. Additional labels, $\mathrm{P}_{2}$ and $\mathrm{S}_{2}$, are added in these figures to identify the direct reflected pulses coming from the second cavity. When the parameter $R$ decreases, the pulses reflected directly from each cavity are better defined, because of the longer travel time differences between the pulses $P_{1}, S_{1}, P_{2}$ and $S_{2}$. However, the amplitude of these pulses $\left(\mathrm{P}_{2}\right.$ and $\left.\mathrm{S}_{2}\right)$ decreases progressively as the size of the second cavity decreases (i.e. as $R$ decreases). Similar behavior is exhibited by the pulses generated by reverberations between the cavities. As the apparent velocity decreases, both the amplitude of the different pulses and their arrival times decrease, as in the previous case (see figures 9b-9d). Furthermore, the scattered field produced by the second cavity loses its importance as the apparent velocity decreases. When the velocity reaches $c=2656 \mathrm{~m} / \mathrm{s}$, the scattered field produced by the second cavities is very weak, and the field generated indicates the existence of guided waves, mainly produced by the first cavity.

Next, to evaluate the scattering produced by the second cavity better, the earlier vertical scattering results are presented in the space-frequency $(x, f)$ domains. Only the scenarios including the largest $(R=10.0 \mathrm{~m})$ and the smallest second cavity $(R=2.5 \mathrm{~m})$, and its absence, were selected as examples. Figure 10 displays these results in a grey-scale format. The amplitude of the scattered field generated when the second cavity is absent is given as a reference, and only the amplitude of the scattered field difference generated when a second cavity is introduced in the medium is shown in figure 10. Four apparent velocities are displayed: $c=\infty \mathrm{m} / \mathrm{s}$, $c=7000 \mathrm{~m} / \mathrm{s}, c=4208 \mathrm{~m} / \mathrm{s}$ and $c=2656 \mathrm{~m} / \mathrm{s}$.

Our results indicate that when one cavity is present the larger reflections appear in the vicinity of the axis of the inclusion, allowing its position to be detected. As the apparent velocity decreases the amplitude of the scattered field diminishes, mainly for higher frequencies. When two cavities are present, the amplitude of the scattered field is slightly modified. However, its difference, as displayed in figure 10, permits the detection of 


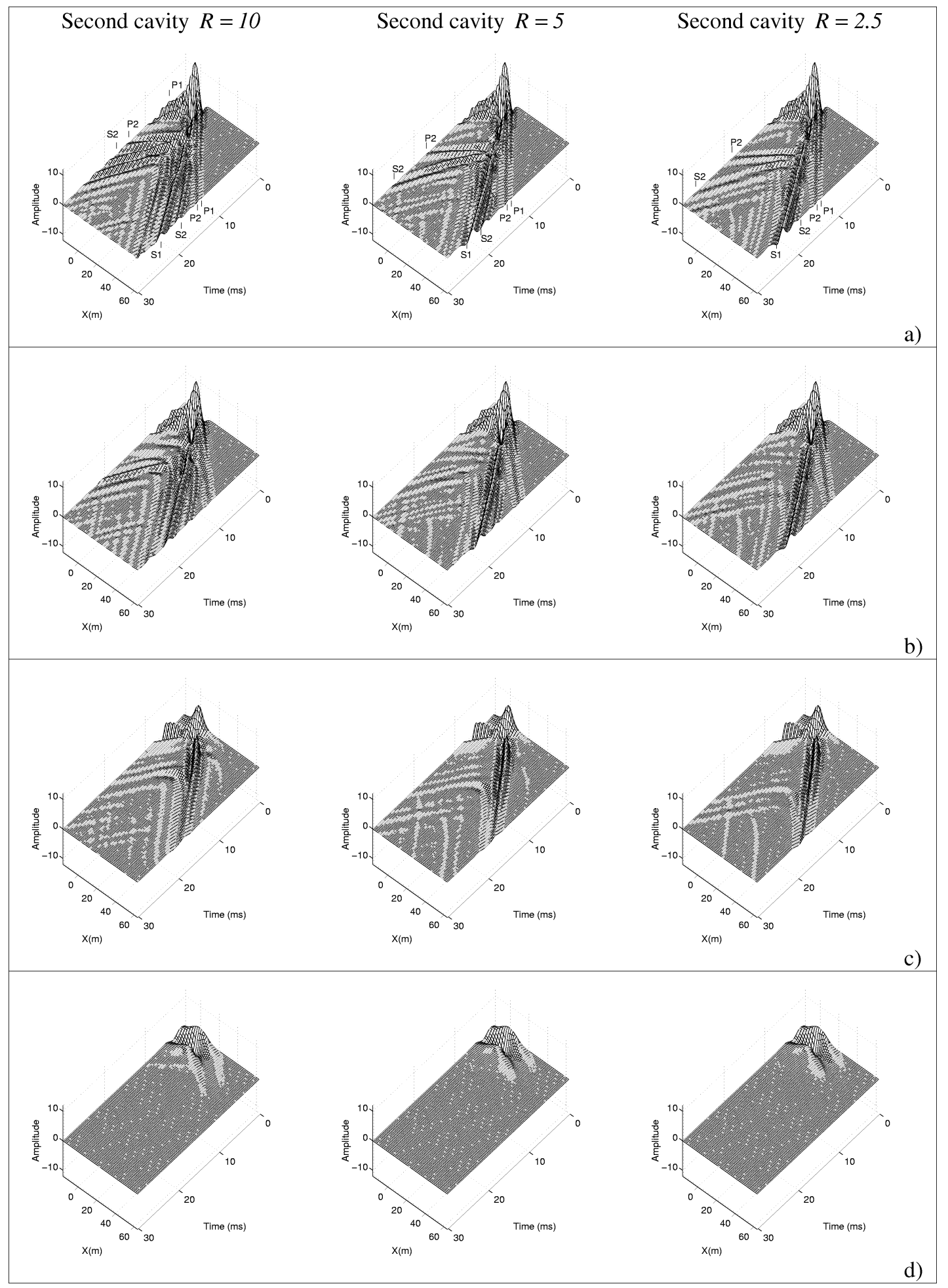

Figure 9. Synthetic seismograms when two cavities are present: (a) $c=\infty \mathrm{m} / \mathrm{s} ;$ (b) $c=7000 \mathrm{~m} / \mathrm{s}$; (c) $c=4208 \mathrm{~m} / \mathrm{s}$; (d) $c=2656 \mathrm{~m} / \mathrm{s}$. 

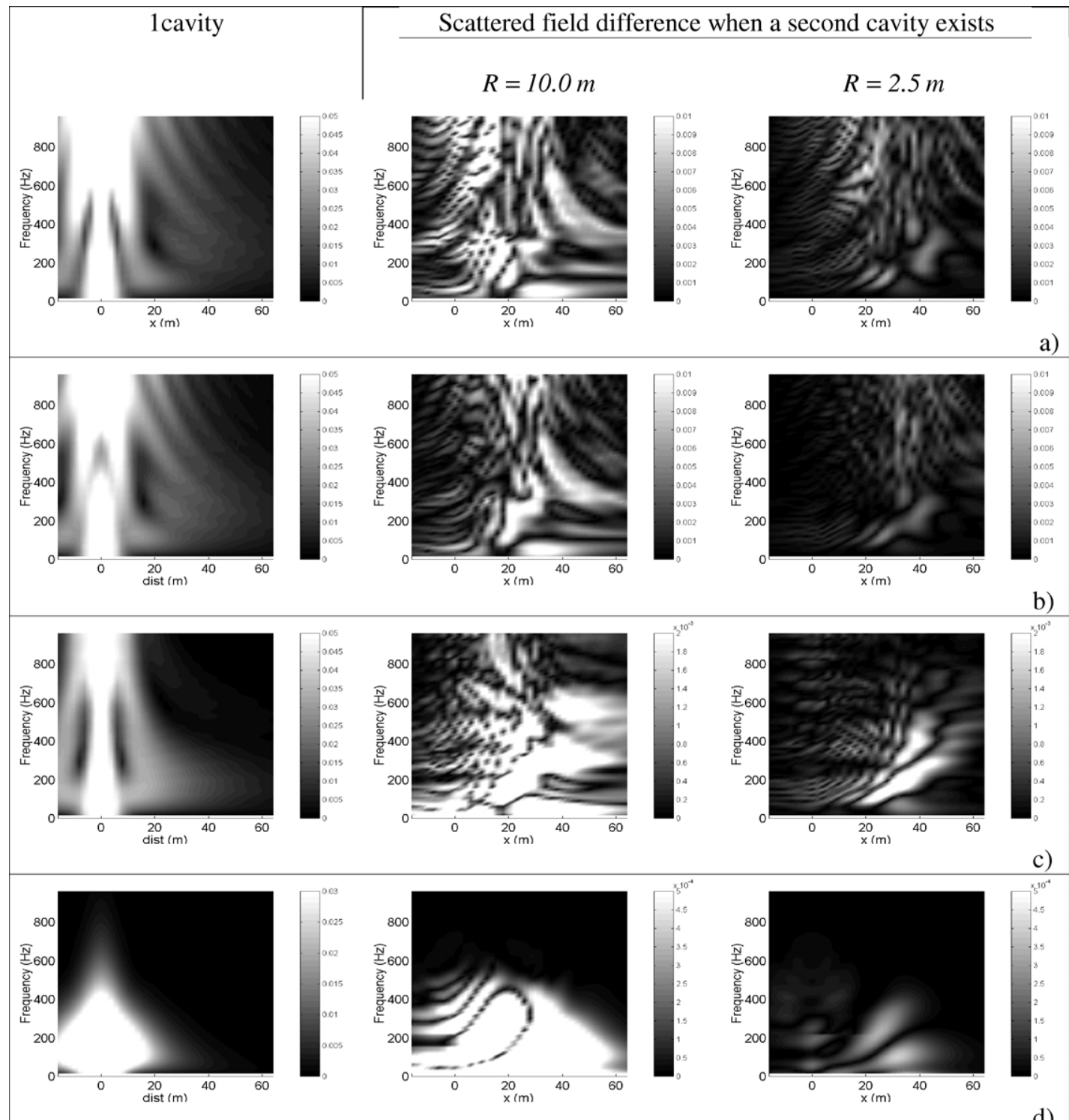

Figure 10. Spectral amplitude of the vertical displacements: (a) $c=\infty \mathrm{m} / \mathrm{s}$; (b) $c=7000 \mathrm{~m} / \mathrm{s}$; (c) $c=4208 \mathrm{~m} / \mathrm{s}$; (d) $c=2656 \mathrm{~m} / \mathrm{s}$.

higher reflections in the vicinity of the second cavity axis, even when the size of second cavity decreases. In these plots, the presence of the second cavity is more visible at lower frequencies. As the apparent velocity decreases the amplitude of the scattered field difference diminishes.

Another interesting aspect of this problem is the creation of shadow zones behind the cavities (Tadeu et al., 1996). Figure 11 illustrates this phenomenon. It shows the amplitude of the total vertical displacements in the surrounding medium, produced by a harmonically vibrating source at position $\mathrm{O}$, when one and two cavities of $R=10.0 \mathrm{~m}$ are present. The total responses are shown as three dimensional plots for two values of the excitation frequency, namely, $200 \mathrm{~Hz}$ and $600 \mathrm{~Hz}$, which correspond to incident wavelengths of $21.0 \mathrm{~m}$ and $7.0 \mathrm{~m}$, respectively. The results for $200 \mathrm{~Hz}$ show a slight shadow behind the cavities. As the frequency increases 

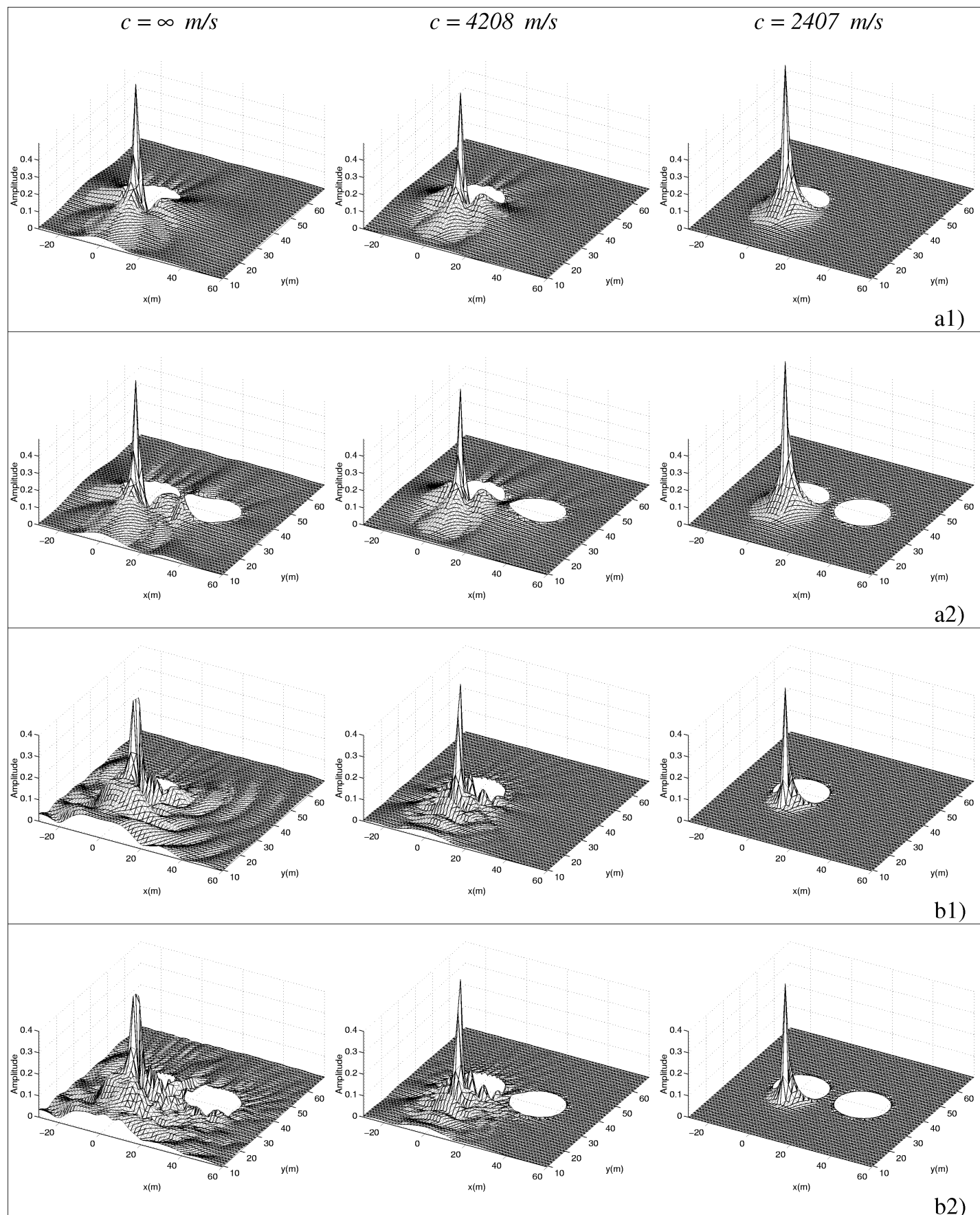

Figure 11. Steady state response. Absolute value of the total vertical displacement fields. (a) excitation frequency $f=200 \mathrm{~Hz}$ : (a1) presence of one oval cavity $R=10.0 \mathrm{~m}$; (a2) presence of two oval cavities $R=10.0 \mathrm{~m}$. (b) Excitation frequency $f=600 \mathrm{~Hz}$ : (b1) presence of one oval cavity $R=10.0 \mathrm{~m}$; (b2) presence of two oval cavities $R=10.0 \mathrm{~m}$. 
to $600 \mathrm{~Hz}$ this shadow becomes more visible. This behaviour was naturally expected, since it is well known that waves whose wavelength is large in comparison to the obstacles they encounter suffer very little scattering. The intense degree of interaction between the cavities, caused by the previously mentioned reverberations, is also clearly visible when $c=\infty \mathrm{m} / \mathrm{s}$, and at a higher frequency of excitation. As already concluded, the presence of the second cavity loses its importance as the apparent velocity decreases. This phenomenon is more pronounced when the excitation frequency increases.

\section{Conclusions}

The boundary element formulation, developed and used to evaluate the 3D scattered field generated by a dilatational point load illuminating cylindrical oval cavities in an unbounded homogeneous elastic medium, was found to be efficient. It could therefore be used in the context of research on seismic tomography problems.

This program was used to compute the wave fields in the vicinity of obstacles (inclusions) embedded in homogeneous elastic media when they are illuminated by dilatational point sources. The simulations performed included the evaluation of the scattered field in the presence of different sized cylindrical oval inclusions.

The frequency and synthetic seismograms were built following waves with different apparent wave velocities along the $z$ axis. The time responses appear to be very complicated, but were consistent with the predictions given by ray acoustics, and were used by the authors to elucidate the most important aspects, with an eye to the development of non-destructive testing and imaging methods.

Our results indicate that the scattered field produced by a second cavity, placed in the vicinity of a cavity nearest to the blast load point, loses its importance as the apparent velocity decreases. Furthermore, the guided waves are mainly produced by the cavity closer to the blast load.

An important finding of this research is that the scattered field difference caused by the introduction of a second cavity makes it possible to identify higher reflections in the vicinity of the second cavity axis, even when the second cavity is small.

\section{Appendix A: The Green's functions}

$\begin{array}{ll}\text { Definitions } & \\ \lambda, \mu & \text { Lamé constants } \\ \rho & \text { Mass density } \\ \alpha=\sqrt{(\lambda+2 \mu) / \rho} & \text { P wave velocity } \\ \beta=\sqrt{\mu / \rho} & \text { S wave velocity } \\ k_{p}=\omega / \alpha, k_{s}=\omega / \beta & \\ k_{\alpha}=\sqrt{k_{p}^{2}-k_{z}^{2}}, k_{\beta}=\sqrt{k_{s}^{2}-k_{z}^{2}} & \\ A=\frac{1}{4 i \rho \omega^{2}} & \text { Amplitude } \\ \gamma_{i}=\frac{\partial r}{\partial x_{i}}=\frac{x_{i}}{r}, i=1,2 & \text { Direction cosines } \\ H_{n \alpha}=H_{n}^{(2)}\left(k_{\alpha} r\right), H_{n \beta}=H_{n}^{(2)}\left(k_{\beta} r\right) & \text { Hankel functions } \\ B_{n}=k_{\beta}^{n} H_{n \beta}-k_{\alpha}^{n} H_{n \alpha} & B_{n} \text { functions }\end{array}$


Green's functions

$$
\begin{gathered}
G_{x x}=A\left[k_{s}^{2} H_{0 \beta}-\frac{1}{r} B_{1}+\gamma_{x}^{2} B_{2}\right], \\
G_{y y}=A\left[k_{s}^{2} H_{0 \beta}-\frac{1}{r} B_{1}+\gamma_{y}^{2} B_{2}\right], \\
G_{z z}=A\left\lfloor k_{s}^{2} H_{0 \beta}-k_{z}^{2} B_{0}\right\rfloor, \\
G_{x y}=G_{y x}=\gamma_{x} \gamma_{y} A B_{2}, \\
G_{x z}=G_{z x}=i k_{z} \gamma_{x} A B_{1}, \\
G_{y z}=G_{z y}=i k_{z} \gamma_{y} A B_{1} .
\end{gathered}
$$

Volumetric strain (super-index $=$ direction of load)

$$
\begin{aligned}
\varepsilon_{\mathrm{Vol}}^{l} & =G_{x l, x}+G_{y l, y}+G_{z l, z} \\
& =A\left[\frac{\partial}{\partial x_{l}}\left(k_{s}^{2} H_{0 \beta}\right)+B_{0, x l x}+B_{0, y l y}+B_{0, z l z}\right] \\
& =A \frac{\partial}{\partial x_{l}}\left[k_{s}^{2} H_{0 \beta}+B_{0, x x}+B_{0, y y}+B_{0, z z}\right] \\
& =A \frac{\partial}{\partial x_{l}}\left[k_{s}^{2} H_{0 \beta}+\hat{\nabla}^{2} B_{0}\right] .
\end{aligned}
$$

Note: $H_{0 \beta, l}=-k_{\beta} \gamma_{l} H_{l \beta}, H_{0 \beta, z}=-i k_{z} H_{0 \beta}$.

Strain components (tensor definition, not engineering)

$$
\begin{aligned}
\varepsilon_{i j}^{l} & =\frac{1}{2}\left(G_{i l, j}+G_{j l, i}\right) \\
& =\frac{1}{2} A\left(\delta_{i l} k_{s}^{2} H_{0 \beta, j}+\delta_{j l} k_{s}^{2} H_{0 \beta, i}+B_{0, i l j}+B_{0, j l i}\right) \\
& =\frac{1}{2} k_{s}^{2} A\left(\delta_{i l} H_{0 \beta, j}+\delta_{j l} H_{0 \beta, i}\right)+A B_{0, i j l} .
\end{aligned}
$$

(a) Strains for loads in the plane, $l=x, y$

$$
\begin{gathered}
\varepsilon_{\mathrm{Vol}}^{l}=\gamma_{l} A\left(-k_{s}^{2} k_{\beta} H_{1 \beta}+k_{z}^{2} B_{1}+\frac{4}{r} B_{2}-B_{3}\right), \\
\varepsilon_{x x}^{l}=\gamma_{l} A\left(\left(\frac{2}{r} B_{2}-k_{s}^{2} k_{\beta} H_{1 \beta}\right) \delta_{x l}+\frac{1}{r} B_{2}-\gamma_{x}^{2} B_{3}\right), \\
\varepsilon_{y y}^{l}=\gamma_{l} A\left(\left(\frac{2}{r} B_{2}-k_{s}^{2} k_{\beta} H_{1 \beta}\right) \delta_{y l}+\frac{1}{r} B_{2}-\gamma_{y}^{2} B_{3}\right),
\end{gathered}
$$




$$
\begin{gathered}
\varepsilon_{z z}^{l}=\gamma_{l} k_{z}^{2} A B_{1}, \\
\varepsilon_{x y}^{l}=A\left(\left(\frac{1}{r} B_{2}-\frac{1}{2} k_{s}^{2} k_{\beta} H_{1 \beta}\right)\left(\delta_{x l} \gamma_{y}+\delta_{y l} \gamma_{x}\right)-\gamma_{x} \gamma_{y} \gamma_{l} B_{3}\right), \\
\varepsilon_{x z}^{l}=i k_{z} A\left(\left(\frac{1}{r} B_{1}-\frac{1}{2} k_{s}^{2} H_{0 \beta}\right) \delta_{x l}-\gamma_{x} \gamma_{l} B_{2}\right), \\
\varepsilon_{y z}^{l}=i k_{z} A\left(\left(\frac{1}{r} B_{1}-\frac{1}{2} k_{s}^{2} H_{0 \beta}\right) \delta_{y l}-\gamma_{y} \gamma_{l} B_{2}\right) .
\end{gathered}
$$

(b) Strain for axial loads, $l=z$

$$
\begin{gathered}
\varepsilon_{\mathrm{Vol}}^{z}=i k_{z} A\left(-k_{s}^{2} H_{0 \beta}+k_{z}^{2} B_{0}+\frac{2}{r} B_{1}-B_{2}\right), \\
\varepsilon_{x x}^{z}=i k_{z} A\left(\frac{1}{r} B_{1}-\gamma_{x}^{2} B_{2}\right) \\
\varepsilon_{y y}^{z}=i k_{z} A\left(\frac{1}{r} B_{1}-\gamma_{y}^{2} B_{2}\right) \\
\varepsilon_{z z}^{z}=i k_{z} A\left(-k_{s}^{2} H_{0 \beta}+k_{z}^{2} B_{0}\right) \\
\varepsilon_{x y}^{z}=-i k_{z} \gamma_{x} \gamma_{y} A B_{2} \\
\varepsilon_{x z}^{z}=\gamma_{x} A\left(-\frac{1}{2} k_{s}^{2} k_{\beta} H_{1 \beta}+k_{z}^{2} B_{1}\right) \\
\varepsilon_{y z}^{z}=\gamma_{y} A\left(-\frac{1}{2} k_{s}^{2} k_{\beta} H_{1 \beta}+k_{z}^{2} B_{1}\right) .
\end{gathered}
$$

Stresses

$$
\tau_{i j}^{l}=\lambda \varepsilon_{\mathrm{Vol}}^{l} \delta_{i j}+2 \mu \varepsilon_{i j}^{l}
$$

\section{References}

Bamberger, A., Joly, P., Kern, M., 1991. Propagation of elastic surface waves along a cylindrical cavity of arbitrary cross section. Mathematical Modelling and Numerical Analysis (M2AN) 25 (1), 1-30.

Banerjee, P.K., 1994. The Boundary Element Methods in Engineering. McGraw-Hill.

Bouchon, M., Aki, K., 1977. Discrete wave-number representation of seismic-source wave field. Bull. Seism. Soc. Am. 67, $259-277$.

Brebbia, C.A., Telles, J.C.F., Wrobel, L.C., 1984. Boundary Elements Techniques. Springer-Verlag.

Datta, S.K., Shah, A.H., 1982. Scattering of SH-waves by embedded cavities. Wave Motion 4, 265-283.

Eringen, A.C., 1961. Propagation of elastic waves generated by dynamical loads on a circular cavity. Journal of Applied Mechanics 28, 218-222.

Kausel E., 1974. Forced vibrations of circular foundations in layered media, MIT Research Report 70-3, Department of Civil Engineering, Massachusetts Institute of Technology, Cambridge, MA.

Kausel, E., Roesset, J.M., 1992. Frequency domain analysis of undamped systems. Journal of Engineering Mechanics ASCE 118 (4), $721-734$.

Lee, V.W., 1977. On deformations near circular underground cavity subjected to incident plane SH waves, in: Symp. of Appl. Computer Methods in Eng., Univ. of Southern California, Los Angeles, pp. 951-961. 
Lee, V.W., 1988. Three-dimensional diffraction of elastic waves by a spherical cavity in an elastic half-space. 1: Closed-form solutions. Soil Dyn. Earthq. Eng. 7, 149-161.

Lee, V.W., Karl, J.A., 1992. Diffraction of SV waves by underground circular cylindrical cavities. Soil Dyn. Earthq. Eng. 11, 445-456.

Lee, V.W., Wu, X., 1994. Application of the weighted residual method to diffraction by 2-D canyons of arbitrary shape: II. Incident P, SV and Rayleigh waves. Soil Dyn. Earthq. Eng. 13, 365-375.

Lysmer, J., Udaka, T., Seed, H.B., Hwang, R., 1974. LUSH - A computer program for complex response analysis of soil-structure systems, Report No. EERC 74-4, Earthquake Engineering Research Center, University of California, Berkeley, CA.

Manolis, G.D., Beskos, D.E., 1988. Boundary Element Methods in Elastodynamics. Unwin Hyman, London.

Moeen-Vaziri, N., Trifunac, M.D., 1988. Scattering and diffraction of plane P and SV waves by two-dimensional inhomogeneities: Part II. Soil Dyn. Earthq. Eng. 7, 189-200.

Ohtsuki, A., Harumi, K., 1983. Effect of topography and subsurface inhomogeneities on seismic SV waves. Earthq Eng. Struct. Dyn. 11, 441-462. Pao, Y.H., Mow, C.C., 1973. Diffraction of Elastic Waves and Dynamic Stress Concentrations. Crane and Russak, New York.

Pedersen, H.A., Sánchez-Sesma, F.J., Campillo, M., 1994. Three-dimensional scattering by two-dimensional topographies. Bulletin of the Seismological Society of America 84, 1169-1183.

Phinney, R.A., 1965. Theoretical calculation of the spectrum of first arrivals in layered elastic mediums. J. Geophys. Res. 70, 5107-5123.

Sanchez-Sesma, F.J., 1983. Diffraction of elastic waves by three dimensional surface irregularities. Bull. Seism. Soc. Am. 73, 1621-1636.

Shah, A.H., Wong, K.C., Datta, S.K., 1982. Diffraction of plane SH waves in a half-space. Earthq. Eng. and Struct. Dyn. 10, 519-528.

Stamos, A.A., Beskos, D.E., 1996. 3-D seismic response analysis of long lined tunnels in half-space. Journal of Soil Dynamics and Earthquake Engineering 15, 111-118.

Tadeu, A.J.B., Santos, P.F.A., Kausel, E., 1999-a. Closed-form integration of singular terms for constant, linear and quadratic boundary elements Part I: SH wave propagation. EABE - Engineering Analysis with Boundary Elements 23 (8), 671-681.

Tadeu, A.J.B., Santos, P.F.A., Kausel, E., 1999-b. Closed-form integration of singular terms for constant, linear and quadratic boundary elements Part II: SV-P wave propagation. EABE - Engineering Analysis with Boundary Elements 23 (9), 757-768.

Tadeu, A.J.B., Kausel, E., Vrettos, C., 1996. Scattering of waves by subterranean structures via the boundary element method. Journal of Soil Dynamics and Earthquake Engineering 15 (6), 387-397.

Trifunac, M.D., 1971. Surface motion of a semi-cylindrical alluvial valley for incident plane SH waves. Bull. Seism. Soc. Am. 61, $1755-1770$.

Waas, G., 1972. Linear two-dimensional analysis of soil dynamics problems in semi-infinite layered media. PhD dissertation. University of California, Berkeley, CA.

Wong, H.L., Trifunac, M.D., 1974. Surface motion of semi-elliptical alluvial valley for incident plane SH-waves. Bull. Seism. Soc. Am. 64, 13891403 . 